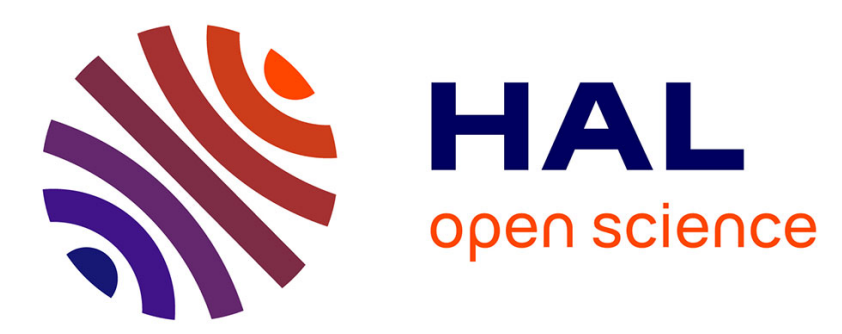

\title{
L'émergence d'un nouveau mode de gouvernement local des réseaux en Allemagne. Une ville moyenne et son Stadtwerk face au processus de libéralisation
}

\author{
Adrien Fender, François-Mathieu Poupeau
}

\section{- To cite this version:}

Adrien Fender, François-Mathieu Poupeau. L'émergence d'un nouveau mode de gouvernement local des réseaux en Allemagne. Une ville moyenne et son Stadtwerk face au processus de libéralisation. Sociologie du Travail, 2007, 49 (4), pp.366-382. halshs-00309046

\section{HAL Id: halshs-00309046 \\ https://shs.hal.science/halshs-00309046}

Submitted on 5 Aug 2008

HAL is a multi-disciplinary open access archive for the deposit and dissemination of scientific research documents, whether they are published or not. The documents may come from teaching and research institutions in France or abroad, or from public or private research centers.
L'archive ouverte pluridisciplinaire HAL, est destinée au dépôt et à la diffusion de documents scientifiques de niveau recherche, publiés ou non, émanant des établissements d'enseignement et de recherche français ou étrangers, des laboratoires publics ou privés. 
« L'émergence d'un nouveau mode de gouvernement local des réseaux en Allemagne.

Une ville moyenne et son Stadtwerk face au processus de libéralisation » Adrien Fender, François-Mathieu Poupeau*

\section{Résumé}

A partir de l'étude d'un cas dans la longue durée, celui d'un Stadtwerk situé dans une ville moyenne, cet article s'interroge sur la recomposition du mode de gouvernement local des réseaux en Allemagne. Il montre comment les réformes néo-libérales et la crise des finances publiques communales constituent de puissants facteurs de transformation. Celle-ci se traduit non pas par une perte de pouvoir pure et simple pour la ville mais par une profonde reconfiguration des relations qu'elle entretenait traditionnellement avec son Stadtwerk. Un nouveau type d'interaction émerge, qui repose sur une plus grande formalisation et sur une certaine "mise à distance " du politique, la ville se réappropriant de son côté une fonction de pilotage stratégique et financier que la période précédente, celle du monopole et de la prospérité communale, avait tendance à minorer.

Mots-clés : Stadtwerk; villes ; réseaux; libéralisation; gouvernance urbaine ; action publique

\section{Introduction}

Avec la diffusion des réformes néo-libérales sur l'ensemble du continent européen, on assiste aujourd'hui à une profonde recomposition des modèles nationaux de service public. Si la France figure en bonne place parmi les pays où les débats ont été particulièrement vifs, l'Allemagne a été elle aussi sujette à de nombreuses interrogations. Les directives adoptées par l'Union européenne dans les domaines de l'énergie ou de l'eau touchent en effet à une tradition d'intervention publique séculaire et qui a longtemps fait l'objet d'un large consensus au sein de la société allemande. Contrairement aux modèles français et anglo-saxons, cette intervention repose sur deux principes forts, qui sont aujourd'hui battus en brèche par les réformateurs (Muschen, 1993 ; Marcou, 1995 ; Lorrain, 2002a; Wollmann, 2002). Le premier est de conférer aux communes un rôle central dans la fourniture des services publics en réseau, via, notamment, les entreprises municipales (Stadtwerke) qui ont été créées à partir du dix-neuvième siècle. Fortes des prérogatives que leur accorde le droit constitutionnel, les communes ont ainsi pris en charge, au fur et à mesure que s'exprimaient de nouveaux besoins, des secteurs aussi variés que l'énergie, l'eau,

\footnotetext{
* Adrien Fender est titulaire d'un DEA de Sociologie (IEP Paris). François-Mathieu Poupeau est chargé de recherches CNRS au Laboratoire Techniques, Territoires et Sociétés (Université Paris Est). Pour toute correspondance, F.-M. Poupeau, LATTS-ENPC, Cité Descartes, 6-8 av. Blaise Pascal, 77455 Marne-la-Vallée cedex 2 (fm.poupeau@enpc.fr)
} 
l'assainissement ou les transports, devenant par là même des vecteurs de modernité et de solidarité. Le second principe est de mettre en avant une forte intégration horizontale entre ces différentes activités. Ainsi donc, contre une conception défendant une vision sectorielle et verticale de la gestion du service public (conception française par exemple), les communes allemandes ont mis en place, par touches successives, un système très intégré localement, qui repose sur la mutualisation des charges entre secteurs et sur la possibilité d'en subventionner certains, structurellement déficitaires (transports), par les bénéfices dégagés par d'autres (énergie, eau).

Jugé par ses détracteurs comme étant opaque et peu efficace, ce mode de gestion est aujourd'hui mis en difficulté. La déstabilisation provient en premier lieu des réformes qui se sont attachées, depuis le début des années 1990, à introduire une concurrence dans les activités organisées en réseaux. S'attaquant essentiellement au secteur énergétique, qui constitue la pierre angulaire du modèle allemand, ces réformes menacent de remettre en question l'édifice construit par les collectivités locales allemandes tout au long du vingtième siècle (Courivaud, 2000 ; Lorrain, 2002a; Steckert, 2002 ; Wollmann, 2002). L'autre facteur de perturbation est à rechercher dans la situation de tension financière que connaissent aujourd'hui les communes allemandes. Devant faire face à de fortes tensions budgétaires, bon nombre de villes cherchent aujourd'hui de nouvelles ressources. Sollicitées par les opérateurs privées, allemands ou étrangers, elles peuvent être tentées de revoir leurs relations avec leurs Stadtwerke, qui sont sources de rentrées financières non négligeables.

L'ensemble de ces mutations suscitent aujourd'hui de nombreuses questions quant à la capacité des communes à demeurer des acteurs importants du modèle allemand de service public. Contraintes, pour beaucoup, à vendre tout ou partie du capital qu'elles possèdent dans les Stadtwerke pour assainir leur budget, celles-ci sont parfois présentées comme les perdantes potentielles des réformes, au profit des grands opérateurs et d'un Etat fédéral qui n'a cessé d'observer avec critique le système mis en place ces dernières décennies (Courivaud, 2000; Wollmann, 2002). Sans minorer l'ampleur des changements pour les communes, il nous semble cependant qu'il se joue autre chose qu'une perte pure et simple d'autonomie ou de capacité d'action locales dans les transformations actuelles. A travers elles, c'est aussi et surtout un nouveau rapport entre la puissance publique locale et les opérateurs de réseaux qui se donne à voir, une nouvelle façon pour les villes de réguler des activités hautement importantes pour leurs élus et leur population. Afin d'illustrer la nature de cette refondation et des transformations qui y sont sous-jacentes, nous prendrons l'exemple de la ville d'Ikstadt et de son Stadtwerk ERTI ${ }^{1}$. Métropole régionale de 130.000 habitants située dans un Land

\footnotetext{
${ }^{1}$ cet article repose sur une enquête de terrain de plusieurs mois, durant laquelle une soixantaine d'entretiens ont été menés, ainsi que la consultation de documents internes et externes (revue de presse notamment) au Stadtwerk. Pour des raisons de confidentialité, nous utiliserons des pseudonymes pour évoquer notre cas d'étude. ERTI est l'acronyme d'Entreprise de Réseaux et de Transports d'Ikstadt, la holding qui coordonne l'ensemble des Stadtwerke d'Ikstadt
} 
riche, Ikstadt représente un cas tout à fait significatif de la voie médiane que la plupart des communes allemandes ont suivie depuis la libéralisation. Fondé depuis plus d'un siècle, son Stadtwerk, qui gère plusieurs activités, dont l'énergie, l'eau et les transports, n'a pas été cédé en totalité à un énergéticien national ou étranger. Désirant, certes, renflouer les caisses de la municipalité mais également conserver un contrôle sur leur opérateur, les élus ont opté pour une solution intermédiaire, à savoir vendre une partie du capital à des opérateurs régionaux, eux-mêmes rachetés par la suite par E-On, un grand énergéticien national. De par sa taille, sa stratégie de modernisation et non de rupture, Ikstadt constitue donc un laboratoire intéressant pour saisir les mutations à l'œuvre dans le rôle de la puissance publique locale.

Après avoir montré, dans une première partie, comment s'exerçait la tutelle de la ville sur ERTI à la veille de la libéralisation (1), nous aborderons la période contemporaine en mettant en évidence comment les transformations récentes contribuent à reconfigurer les relations entre les deux partenaires (2). Cette refondation fait apparaître un double mouvement, de nature dialectique. Elle va tout d'abord dans le sens d'une plus grande «mise à distance du politique » dans la gestion quotidienne du Stadtwerk, qui donne à ce dernier une plus grande latitude dans la conduite de son activité. Cette "mise à distance » n'est pas cependant synonyme d'éviction totale du politique. Dans le même temps en effet, la ville se réapproprie une fonction de pilotage stratégique et financier que la période précédente, celle du monopole et de la prospérité communale, avait tendance à minorer. Avec cette transformation de la nature de la tutelle politique sur ERTI, c'est une nouvelle forme de gouvernement local du modèle allemand de service public qui se donne à voir, qui confère une place de plus en plus importante au maire et à une nouvelle génération d'élus et de fonctionnaires au cœur de la diffusion du New Public Management.

\section{ERTI, un partenaire central pour la ville d'Ikstadt}

\subsection{Du Referat à la GmbH, la constitution d'un acteur majeur dans la ville}

Comme beaucoup de ses homologues, ERTI s'est constituée par touches successives, au fur et à mesure que de nouveaux besoins ont été exprimés et relayés par la population et les élus locaux. En 1855, le gaz et l'eau sont les premiers réseaux de service public à susciter l'intérêt de la ville. Ne trouvant guère de partenaire privé pour répondre à ses attentes, la ville décide de prendre en main elle-même la construction d'une usine à gaz et d'un réseau public destiné à fournir de l'éclairage aux rues mais aussi aux particuliers. L'électricité quant à elle ne fait son apparition qu'à la toute fin du dix-neuvième siècle. En 1899, après deux ans de travaux, une centrale électrique est construite. Commence alors une phase intense d'électrification de la ville, qui se poursuit jusqu'à la veille de la seconde guerre mondiale. Là encore, comme pour le gaz et l'eau, c'est la ville qui joue un rôle d'impulsion majeure, finançant la construction d'une seconde 
centrale (1910) puis le développement des réseaux électriques. S'il est fait, par la suite, appel à des fournisseurs d'électricité extérieurs pour subvenir à l'accroissement de la consommation de la population, les élus se refusent à confier l'activité de distribution d'électricité à un acteur privé, préférant en garder le contrôle.

Portée par des besoins importants en matière d'investissement, l'extension du modèle de gestion locale se poursuit avec vigueur tout au long du vingtième siècle. Arrivés, dans leur développement, à une certaine maturité, les premiers réseaux de service public contribuent en partie à financer les nouvelles activités. En 1924, sous la forme, cette fois, d'une société privée, une entreprise de transport est créée pour assurer un service régulier par bus puis tramway. En 1952, la ville lance un réseau de chauffage urbain, alimenté en partie par la centrale fournissant de l'électricité, couplage qui permet de réaliser d'importantes économies.

1965 marque une étape importante dans le développement du modèle de gestion publique locale d'Ikstadt. Jusqu'alors, excepté les transports, l'ensemble des réseaux de gaz, d'eau, d'électricité, de chauffage urbain étaient directement placés dans le giron de la ville, sous la forme d'un département administratif (Referat). En 1965, les élus décident de dissocier le département «Eau et Energie » des services municipaux et d'intégrer ces activités à une holding nouvellement créée pour la circonstance: ERTI. Entreprise privée détenue à $100 \%$ par la ville d'Ikstadt, ERTI regroupe alors deux filiales :

Fig. 1. Organigramme d'ERTI avant la libéralisation

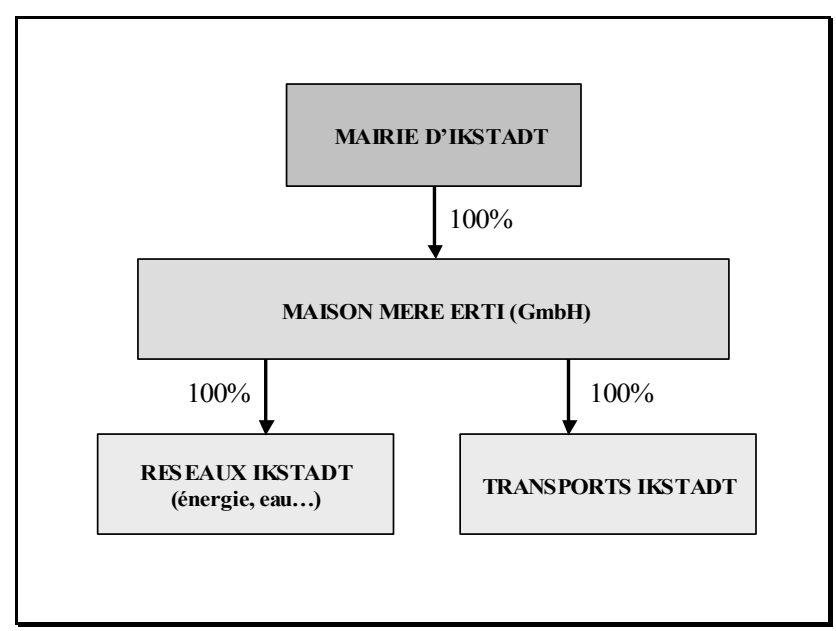

La raison principale incitant la mairie à « démunicipaliser» la prise en charge des réseaux tient aux vertus prêtées au mode de gestion privé, censé favoriser un accès à des capacités de financement plus diversifiées et apporter une plus grande flexibilité de gestion. Le seul inconvénient majeur de cette remise en question des anciens Referat réside dans la nécessité de payer désormais des impôts sur les sociétés, ce qui vient grever les redistributions financières entre activités. Cette 
nouvelle contrainte reste toutefois limitée, grâce au Querverbund, disposition fiscale qui permet à une entreprise privée assurant des missions de service public de compenser les pertes et gains de ses différentes filiales ex ante, c'est-à-dire avant paiement des impôts sur le bénéfice. Dans la pratique, ce sont les pertes de l'activité «transports », structurellement déficitaire depuis la création de cette branche (ou section, Sparte), qui sont compensées par les gains de l'activité réseaux.

Renforcée par ce dispositif fiscal, disposant d'une plus grande latitude d'action, ERTI ne cesse alors d'accroître son champ d'intervention. En 1969, elle reprend dans son giron les activités portuaires qui étaient restées depuis leur création (1904) en régie directe. En 1988, 1993 et 1995, des entreprises de gestion du parc de stationnement, de recyclage et de compost complètent le dispositif. Résultat de cette accumulation de compétences, ERTI se présente ainsi comme un acteur majeur dans la vie politique, économique et sociale de la ville, au moment où s'esquisse le processus de libéralisation. Quelques chiffres et éléments peuvent nous donner un premier aperçu de cette importance ${ }^{2}$. Avec 1135 employés en 2002 et un chiffre d'affaires de 215 M€, ERTI représente le troisième employeur de la ville, derrière les services municipaux et surtout l'université, qui accueille plus de 20.000 étudiants chaque année. Elle est la première entreprise privée de l'agglomération, dont le tissu économique est surtout constitué de petites PMEPMI et d'une brasserie de taille un peu plus importante mais qui alimente un marché essentiellement régional. L'importance d'ERTI pour la ville va toutefois bien au-delà de son seul poids économique. Elle se mesure également à l'aune des transferts financiers que l'entreprise consent chaque année à la ville et qui se composent d'une redevance de concession (Konzessionsabgabe, 10,7 M€ en 2002), de la compensation du déficit récurrent des transports (17 M€) et enfin de la redistribution d'une partie des bénéfices générés par ERTI (2 M€). Rapporté au budget annuel (240 M€ en 2002) et au déficit de la ville (15 M€), ces chiffres permettent d'apprécier l'importance que peut revêtir ERTI pour les élus locaux, surtout dans le contexte de tension financière qui s'instaure à partir du début des années 1990.

\subsection{Au-delà de la tutelle : une relation de très forte proximité}

Du fait de cette histoire particulière, la relation qui lie le Stadtwerk d'Ikstadt à la ville est très étroite jusqu'à la veille de la libéralisation. L'abandon en $1965 \mathrm{du}$ statut de Referat pour celui de GmbH (Gesellschaft mit beschränkter Haftung ${ }^{3}$ ), s'il a desserré certaines contraintes administratives, n'a pas fondamentalement modifié l'emprise de la ville. Même plus libre statutairement, le nouveau Stadtwerk fait toujours fonction de «bras armé » de la ville, certes moins lié organiquement à elle, mais toujours très encadré par des élus et fonctionnaires

\footnotetext{
${ }^{2}$ pour des chiffres plus détaillés concernant ERTI et ses différentes branches, cf l'annexe

3 équivalent d'une Société à responsabilité limitée française
} 
territoriaux soucieux de maîtriser un outil précieux pour leurs politiques publiques.

Cette présence du politique se manifeste avant tout à travers un contrôle étroit et permanent du Stadtwerk, facilité par les fonctions formellement dévolues à la ville. En tant qu'actionnaire de la structure, celle-ci est très présente dans différentes instances de pilotage, en particulier dans les conseils de surveillance (Aufsichtsräte) des sociétés qui composent ERTI. De ce fait, la plupart des grandes orientations stratégiques (diversification vers d'autres secteurs, politique d'investissements...) et des actes de gestion quotidienne de l'entreprise passent sous le regard tatillon des services financiers de l'administration municipale, dans une logique de contrôle ex ante. En tant qu'autorité concédante des activités de réseaux, qui sont le cœur de métier du Stadtwerk, la ville exerce une autre forme de tutelle, complémentaire. Elle porte sur les grandes décisions qui rythment la vie de chacun des services concédés : politique commerciale (tarification...), choix d'investissement... Ce contrôle fait intervenir le conseil municipal, qui, pour une activité comme l'énergie, tient à conserver ses prérogatives en matière de fixation des tarifs de l'électricité, du gaz et des réseaux de chaleur.

Cette tutelle n'est cependant pas uniforme. Selon les périodes et les enjeux politiques qui leur sont associés, les branches du Stadtwerk font l'objet d'un encadrement plus ou moins important. Secteurs particulièrement sensibles durant la première moitié du vingtième siècle, le gaz et l'électricité suscitent moins d'intérêt dans les années qui suivent, au fur et à mesure qu'ils deviennent des produits banalisés, auxquels l'ensemble de la population a accès. Les questions de desserte deviennent moins prégnantes, même si, ponctuellement, certaines situations peuvent donner lieu à un retour du politique. L'énergie est dès lors relayée par l'adduction d'eau puis, à partir des années 1970, par les transports, qui deviennent un enjeu politique majeur pour la municipalité, et ce d'autant plus que la majorité au pouvoir (SPD) doit composer dans les années 1980 avec le parti écologiste. Les demandes politiques se multiplient pour créer de nouvelles lignes ou améliorer la qualité du service. Peu fondées sur une logique de rentabilité mais plus sur une volonté politique de transformation de la vie locale (amélioration du cadre de vie, lutte contre la pollution...), elles mettent le Stadtwerk dans une position où il n'a guère les moyens de s'opposer aux desiderata des élus car jouissant d'un monopole sur l'ensemble des activités qui lui sont concédées. Particulièrement exposé aux demandes sociales, ce secteur n'a donc pas le même rapport avec la ville que l'énergie ou l'adduction d'eau, pour lesquelles la relation au politique est plus distendue.

Les interactions entre ville et Stadtwerk ne sauraient cependant être réduites à cette relation tutélaire définie par le cadre de la concession et par la position d'actionnaire. Au quotidien, l'influence des politiques et fonctionnaires territoriaux s'exerce bien au-delà de ces échanges formalisés, parfois à la limite de la légalité. Si les affaires de corruption, qui ont pu défrayer la chronique dans certaines villes allemandes, n'ont, semble-t-il, pas touché Ikstadt, certaines 
demandes de services au profit de la ville dépassent l'objet social du Stadtwerk et, partant, sont susceptibles de tomber sous les fourches caudines de l'administration fiscale. Considéré comme étant riche, le Stadtwerk est régulièrement sollicité pour résoudre certains problèmes qui se posent à la ville. On lui demande de participer à la construction d'un pont, d'être soucieux d'intégrer ses ouvrages dans la ville ou, bien souvent, de financer la réfection d'une rue qui ne serait pourtant pas à sa charge. Il est également sollicité pour contribuer à l'animation de la vie locale et au rayonnement de la ville, en devenant sponsor d'un club sportif ou en agissant comme ambassadeur de la ville à l'étranger. L'ensemble de ces interactions, qui se font sous une surveillance lâche de l'administration fiscale et de l'Office des cartels renforcent le lien étroit qui unit la ville à son Stadtwerk qui, bien que n'étant plus Referat, reste toujours sous contrôle.

\section{Une tutelle bien acceptée par le Stadtwerk car source de protection et d'autonomie}

Malgré les contraintes qu'il induit, ce comportement de la ville, qui n'est pas sans rappeler l'attitude de l'Etat français à l'égard des entreprises nationalisées ${ }^{4}, \mathrm{n}$ 'est pas si mal vécu par le Stadtwerk, et ce pour deux principales raisons. La première est que, tout en cultivant sa différence vis-à-vis des services de la ville, perçus comme une bureaucratie de fonctionnaires assez peu soucieux de l'usager, l'ensemble du corps social du Stadtwerk se pense encore comme une excroissance de la municipalité. Les traces laissées dans la culture interne par l'ancien statut de Referat, l'existence, à tout niveau hiérarchique, de liens très forts entre agents des Stadtwerke et fonctionnaires de la ville, renforcent le sentiment d'appartenance à une même communauté, celle d'Ikstadt. Elles rendent plus acceptables les prestations de services dérogatoires, considérées par bien des individus comme une contrepartie légitime, qui profite à la ville toute entière.

La seconde raison est que le Stadtwerk n'est pas dénué de moyens d'action sur sa tutelle et qu'en contrepartie de sa relative perméabilité aux demandes politiques, il jouit d'une forme de protection, qui lui confère un certain confort en interne. D'une part, la municipalité n'abuse pas de sa position d'actionnaire. Evoluant dans un contexte institutionnel et économique qui, à la veille de la réunification, la soumet à une faible pression financière, elle se contente d'encaisser les redevances de concession et d'occupation du domaine public sans réclamer la participation aux bénéfices qu'elle pourrait légitimement exiger. Elle demande simplement au Stadtwerk qu'il couvre le déficit récurrent et structurel de l'activité de transports avec les bénéfices générés par les autres activités plus rentables. D'autre part, sa qualité d'autorité concédante n'en fait pas un décideur aveugle et indifférent aux revendications de son concessionnaire. Véritable «Etat dans l'Etat » car, nous l'avons souligné, gros employeur sur un territoire qui ne compte pas de véritable tissu industriel, ERTI est un acteur avec lequel élus et

\footnotetext{
${ }^{4}$ pour lequel on a pu parler d' « Etat prédateur » (Cohen, 1992)
} 
fonctionnaires doivent composer. A certaines périodes, il peut s'impliquer dans un scrutin électoral en désavouant ou en s'abstenant de soutenir un candidat suspecté de vouloir introduire une plus forte pression gestionnaire ${ }^{5}$. Instrument d'amélioration du niveau de vie locale, de rayonnement de la ville et de flexibilité dans l'action publique, il est aussi un outil que la ville entend ménager. Enfin, quoique très présents dans la gestion quotidienne de l'entreprise, les services municipaux et les élus ne sont pas toujours aptes à développer une expertise propre. Ils se reposent assez largement sur les documents produits par les services de leur concessionnaire, s'efforçant de détecter les seuils critiques à partir desquels ceux-ci abuseraient de leur situation de monopole. A une vision des rapports entre ville et Stadtwerk en terme de tutelle, simplement médiatisés par des dispositifs juridiques, financiers et contractuels, il faut donc opposer une forme d'interaction davantage fondée sur la négociation informelle, chacune des parties étant soucieuse de ne pas déstabiliser son partenaire par des demandes trop disproportionnées. Cette connivence, qui repose sur une forte connaissance mutuelle et sur une grande confiance, permet la prise en compte en amont des intérêts de l'autre partie. Elle explique notamment que la ville ne se soit jamais de facto opposée aux hausses tarifaires d'électricité demandées par les dirigeants du Stadtwerk "réseaux » et qu'elle ait toujours eu le souci de ne pas trop faire pression sur lui.

\section{Le couple " maire-direction" au centre d'un système d'interaction qui met à distance la holding financière}

Dans ce mode de régulation subtil, qui confère une position très périphérique aux usagers, le couple formé par le maire et les deux dirigeants du Stadtwerk joue un rôle central ${ }^{6}$. Occupant une position classique de «marginaux sécants » (Jamous, 1967), ces acteurs se font à la fois les porte-parole de leur institution vis-à-vis de l'autre partie et les relais des contraintes de celle-ci vis-à-vis de leur propre organisation. Maire sensible aux logiques technico-économiques, dirigeants du Stadtwerk perméables aux demandes des politiques, la confusion des genres facilite la recherche d'un compromis acceptable par tous. La vision globale que les uns et les autres ont de la situation, qui est facilitée par leur position ${ }^{7}$, leur permet d'avoir une capacité à négocier et à accéder à certaines demandes. Porteur des exigences de la ville, notamment sur les questions de transport, le maire exerce au sein de la municipalité un rôle d'arbitrage qui est facilité par le caractère mayoral du gouvernement local et la discipline qui existe au sein de l'équipe

\footnotetext{
${ }^{5}$ ce qui a été le cas en 2002, lors des dernières élections municipales, à l'occasion desquelles l'un des deux directeurs a soutenu le maire en place, ce qui lui a valu de vives critiques de la part de la candidate CDU-CSU, qui sera finalement élue

${ }^{6}$ ERTI a la particularité d'être gérée à la fois par un directeur technique et un directeur commercial ${ }^{7}$ le maire préside l'Aufsichtrat, dirige le conseil municipal et a donc une connaissance large de la situation de la ville et du Stadtwerk. De leur côté, les dirigeants du Stadtwerk cumulent une fonction de direction de la holding financière et des différentes branches du Stadtwerk. Ils ont donc eux aussi une vision globale des enjeux qui se posent à l'ensemble de l'entreprise et peuvent donc plus aisément construire des compromis
} 
municipale ${ }^{8}$. Cette centralité au sein de l'appareil politique lui permet de faire preuve d'une grande flexibilité dans ses revendications vis-à-vis du Stadtwerk, en s'arrangeant pour écarter des demandes politiques jugées trop exagérées pour en appuyer d'autres, en échange de contreparties acceptables pour le Stadtwerk. Ce comportement est conforté par l'attitude des dirigeants du Stadtwerk qui, forts de leur connaissance intime des enjeux politiques locaux ${ }^{9}$ et de la position centrale qu'ils occupent dans leur structure, savent relayer en interne les demandes politiques qu'ils auront monnayées dans l'intérêt bien compris de leur entreprise. Ce rôle de «passeurs » est d'autant plus facilité et accepté par les agents d'ERTI qu'en contrepartie, les dirigeants font écran et les préservent contre ce qui pourrait être une intrusion trop importante du politique. Au contraire, en endossant un rôle de filtre, ils permettent aux responsables de branches de se consacrer pleinement aux contraintes opérationnelles.

Les agents des branches sont surtout en relation avec les fonctionnaires municipaux, ce qui n'exclut pas, là non plus, de nombreuses interactions qui sortent du cadre canonique du contrat de concession (Konzessionsvertrag) et se font sur le registre de l'arrangement. La voirie nous en fournit un exemple éclairant. Dans ce domaine, le contrat de concession détermine avec précision la nature des coûts que chacune des deux parties doit supporter, en fonction des types de travaux à effectuer. La définition précise de tous ces cas de figure ne réduit pourtant pas les relations entre les Stadtwerke et le service voirie à des contacts purement épistolaires et impersonnels. Certains dossiers sont tangents et obligent à se rapprocher pour définir une solution commune, qui tienne compte des contraintes de l'autre. Les agents d'ERTI peuvent alors se montrer assez compréhensifs envers leurs collègues de la ville, en prenant en charge certains coûts contestables, et ce d'autant plus qu'ils pourront compter sur eux à d'autres moments, lorsqu'il s'agit d'apprécier la qualité des travaux de réfection des voiries ou d'obtenir des autorisations de creuser dérogatoires aux règles administratives. A un niveau plus opérationnel, c'est donc, là encore, une logique de l'arrangement qui prévaut et qui permet de résoudre la plupart des problèmes quotidiens sans nécessairement en référer aux instances supérieures.

Cette flexibilité est d'autant plus possible que la holding n'occupe qu'une place mineure dans le mode de gouvernement d'ERTI, au profit de branches beaucoup plus puissantes. Le caractère modéré des exigences financières de la ville, le souci de la direction de l'entreprise de préserver sa capacité à obtenir des opérationnels qu'ils mettent en œuvre les arrangements pris avec le maire sapent toute velléité que la holding pourrait avoir à jouer un rôle plus actif dans la gestion d'ERTI. Ainsi protégées, les branches ont tendance à développer une culture du geste

\footnotetext{
${ }^{8}$ pour reprendre la typologie esquissée par Hellmut Wollmann sur les types de gouvernement urbain en Allemagne (Wollmann, 2004), Ikstadt se situe dans un Land ayant favorisé un régime de type « présidentiel », conférant d'importants pouvoirs au maire. Ceci n'est pas le cas dans d'autres Länder, qui ont opté pour des formules reposant sur un mode de décision de nature plus collégiale 9 avant d'occuper leurs fonctions, les deux dirigeants étaient présidents des deux principales factions politiques de la ville. La plupart des anciens directeurs ont suivi ce type de parcours
} 
technique. Tel est le cas de la branche énergie qui, pouvant compter sur une certaine bienveillance des politiques car véritable « vache à lait » de l'entreprise à même de combler le déficit des transports, n'est que faiblement incitée à gagner en productivité ou en rentabilité. Dans ce contexte, la holding se contente de remplir une simple fonction support d'administration et de prestation de services aux branches, sans pouvoir prétendre s'immiscer de manière fine dans la gestion budgétaire.

\section{L'émergence d'un nouveau mode de relations avec la ville}

Relativement stabilisés dans le temps, ces arrangements se voient doublement remis en question avec, d'une part, l'introduction d'une plus grande concurrence dans le système énergétique allemand et, d'autre part, les difficultés financières croissantes rencontrées par la ville d'Ikstadt à partir de la décennie 1990. Toutefois, les changements ne sont pas synonymes de bouleversement pour le moment. Bien loin d'éradiquer purement et simplement le modèle de pilotage des réseaux qu'a pu connaître Ikstadt ces dernières décennies, ils correspondent plutôt à une forme de déplacement des relations d'interdépendance entre la ville et ERTI, laissant une plus grande place à de nouveaux acteurs jusque là relégués à des rôles périphériques, à savoir la holding et les financiers.

\subsection{ERTI pris en étau entre deux contraintes majeures : l'ouverture à la concurrence et la crise financière des communes}

Même si un certain nombre d'évolutions se dessinent dans les autres secteurs gérés par ERTI, l'énergie est, de loin, l'activité la plus directement au cœur des bouleversements du «système Stadtwerk ». Touchant ce qui constitue la pierre angulaire du modèle allemand de service public, la série de réformes qui ne cessent d'affecter ce secteur depuis le début des années 1990 constitue un puissant levier de changement dans la relation qu'entretient ERTI avec la ville.

La libéralisation du marché de l'énergie, un aiguillon plus qu'une remise en cause radicale

Il ne s'agit pas ici de reprendre dans le détail l'ensemble d'un processus qui a déjà été décrit dans plusieurs travaux ${ }^{10}$. Ceux-ci montrent bien la volonté de certains acteurs, au premier rang desquels l'Etat fédéral, de mieux contrôler voire de remettre en question l'importance des Stadtwerke dans l'économie générale du secteur. Fruit de nombreux débats outre-Rhin, la loi EnWG (Energiewirtschaftsgesetz) du 24 avril 1998 constitue la première étape, fondamentale, vers l'émergence d'un nouveau paysage industriel. Texte

\footnotetext{
10 sur le processus communautaire, cf Schmidt, 1997 ; Levi-Faur, 1999. Sur les réformes allemandes, cf. Courivaud, 2000 ; Eising 1999 et Eberlein, 2004
} 
ambitieux, la loi EnWG va, en théorie, beaucoup plus loin que les objectifs fixés par la Commission Européenne, puisqu'elle impose une ouverture immédiate du marché, y compris pour la clientèle des particuliers. Ainsi donc, l'ancienne organisation fondée sur des monopoles territoriaux protégés par des contrats de démarcation (Demarkationsverträge) doit laisser place à un nouveau type d'architecture, accordant une plus grande importance au marché, dans les domaines de la production et de la fourniture de services. Dans le même temps cependant, la portée effective de la concurrence est réduite par un ensemble de dispositions, ce qui témoigne de la volonté des rédacteurs de la loi de 1998 de ne pas trop déstabiliser les forces en présence, notamment sur le plan local. D'une part, la séparation juridique (unbundling) entre activités de production, de transport et de distribution n'est pas immédiate mais doit se faire par palier, sous forme comptable dans un premier temps. D'autre part, même si le caractère exclusif des concessions est abrogé, les communes ne sont pas obligées d'organiser un appel d'offres pour mettre en concurrence leur Stadtwerk. Ces derniers peuvent donc continuer, pour un temps, à exercer la fonction de gestionnaire du réseau de distribution. Enfin, dernière disposition fondamentale, la loi EnWG n'instaure aucune autorité de régulation indépendante. Contrairement à la plupart des pays européens qui ont opté pour cette formule, ce sont les opérateurs, dont les Stadtwerke, qui, jusqu'à l'été $2004^{11}$, fixent eux-mêmes, par voie contractuelle, les tarifs d'accès aux réseaux. Cette stratégie de changement a deux conséquences. Elle permet tout d'abord aux opérateurs de continuer à rémunérer correctement leur fonction de gestionnaires des réseaux, ce qui leur laisse le temps de se préparer à des restructurations internes et externes (Brunekreeft, Keller, 2000). Ensuite, elle atténue la concurrence des nouveaux entrants, ceux-ci devant s'acquitter de tarifs de péages parfois plus élevés qu'ils ne le seraient du fait de l'intervention d'une autorité de régulation. L'ensemble de ces dispositions expliquent en grande partie le caractère assez peu ouvert du marché, quelques années après l'adoption de la loi. Si, dans les premiers temps, une forte baisse des tarifs a pu être constatée, la mise en concurrence réelle des anciens monopoles a été réduite. Dans leur grande majorité, les particuliers ainsi que les petites entreprises se sont assez peu tournés vers la concurrence et conservent leur fournisseur historique.

Si l'ensemble de ces dispositions invite à une lecture quelque peu "continuiste " des réformes, celles-ci s'inscrivant dans un cadre institutionnel qui joue un rôle d'amortisseur (Eberlein, 2000), il n'en reste pas moins qu'à une échelle plus réduite, les transformations sont importantes, en particulier pour les Stadtwerke, qui ont tout à craindre du formidable mouvement de concentration et de rationalisation qui gagne l'ensemble du secteur énergétique. Face aux menaces du marché, ceux-ci réagissent de manière très différente, en fonction notamment du contexte local (Lorrain, 2002b). Si tous ont en commun de s'engager dans des

\footnotetext{
11 en juillet 2004, suite à une directive européenne prônant la création d'autorités de régulation indépendantes, l'Allemagne doit modifier le système en vigueur. Ce changement ne saurait toutefois être interprété comme le prélude à un bouleversement total, l'autorité de régulation ayant encore une capacité d'action réduite
} 
réformes organisationnelles assez similaires, qui visent à développer de nouvelles compétences internes en matière d'achat d'énergie et de gestion commerciale, leur stratégie face à la concurrence obéit à de multiples contingences. Elle dépend, certes, du contexte économique et industriel dans lequel elles évoluent, de la présence d'opérateurs plus ou moins offensifs sur leur territoire mais aussi, très largement, du comportement de la ville partenaire et de sa capacité à faire face à une contrainte qui lui est propre : la crise des finances locales.

La crise financière des communes et le choix d'une ouverture maîtrisée du capital d'ERTI

Alors qu'elles jouissaient, depuis l'après-guerre, d'une certaine prospérité qui leur permettait d'avoir peu d'exigences financières vis-à-vis de leur Statdwerk, les villes allemandes se trouvent confrontées depuis le début des années 1990 à ce qui apparaît comme une grave crise financière, sans doute la plus importante de leur histoire. Celle-ci s'explique par le fossé qui ne cesse de se creuser depuis la réunification entre des ressources, qui ont plutôt tendance à stagner voire à diminuer, et des dépenses, qui suivent un mouvement opposé (Wollmann, 2002). Ce décalage croissant entre les recettes et les charges communales entraîne un déficit budgétaire persistant. Tout en ne connaissant pas la situation quasicatastrophique de certaines de ses homologues, la ville d'Ikstadt ne fait guère exception à la règle. A partir de 1998, son déficit se creuse pour atteindre, nous l'avons vu, 15 M€ en 2002, soit 7\% du budget annuel. Cette dégradation des finances locales a une conséquence directe pour elle : le blocage de ses comptes par décision du Land et l'interdiction d'investir dans des domaines qui ne sont pas indispensables à la bonne marche des affaires communales. La libéralisation ne saurait donc rendre compte à elle seule des transformations qui affectent les Stadtwerke. Dans le cas d'Ikstadt, le facteur "crise financière" constitue un levier tout aussi important.

A bien regarder l'éventail des stratégies suivies par l'ensemble des villes et des Stadtwerke allemandes, trois types d'options s'offrent en théorie à ERTI (Lorrain, 2002b). La première, peu fréquente, consiste à conserver tels quels le statut et le capital du Stadtwerk. La deuxième - souvent choisie par des villes très endettées se traduit par la vente de la majorité voire de la totalité du capital du Stadtwerk à un investisseur privé. Ecartant ces deux premières options, les dirigeants d'ERTI et les élus de la ville optent pour une voie médiane, largement suivie en Allemagne : ouvrir le capital du Stadtwerk tout en demeurant actionnaire majoritaire. En échange d'un apport en nature (leurs réseaux), deux opérateurs régionaux se voient céder $24 \%$ du capital de la branche énergie d'ERTI. Pour ceux-ci, comme pour E-On qui rachète ensuite ces deux énergéticiens, l'entrée au capital d'ERTI répond à une logique d'intégration industrielle. Elle permet d'avoir accès plus aisément à une bonne partie des clients finals et de bénéficier de la notoriété dont jouit ERTI auprès d'eux. Elle permet également de ne pas affronter directement une entreprise très soutenue par ses élus locaux mais 
d'entrer dans une logique de coopération qui participe de la consolidation de leur position sur le marché. De son côté, ERTI ne subit pas l'opération de manière passive mais la soutient activement au contraire car elle y voit deux intérêts. Le premier est d'étendre son périmètre d'action aux communes avoisinantes en récupérant les réseaux jusque là détenus par les deux opérateurs régionaux. $\mathrm{Ce}$ faisant, elle acquiert une taille critique à même d'assurer sa survie dans un marché appelé à devenir de plus en plus concurrentiel. Le second intérêt est de pouvoir diversifier ses sources de financement en faisant rentrer un actionnaire privé plus à même que la ville de financer la rénovation de sa centrale électrique et d'une partie de ses réseaux. En décidant de limiter l'entrée des nouveaux partenaires à moins de $25 \%$, la ville tient toutefois à conserver l'intégralité de la prise de décision, E-On n'accédant pas à la minorité de blocage. La seule contrepartie qu'obtient l'opérateur est financière : l'assurance de toucher de la part de la branche énergie un montant minimal de dividendes, et ce avant compensation du déficit par l'activité de transport public. Cette nouvelle participation au capital de l'entreprise occasionne quelques bouleversements au sein d'ERTI, qui se dote d'une nouvelle structure, beaucoup plus complexe que la précédente :

Fig. 2. L'organisation d'ERTI après la libéralisation

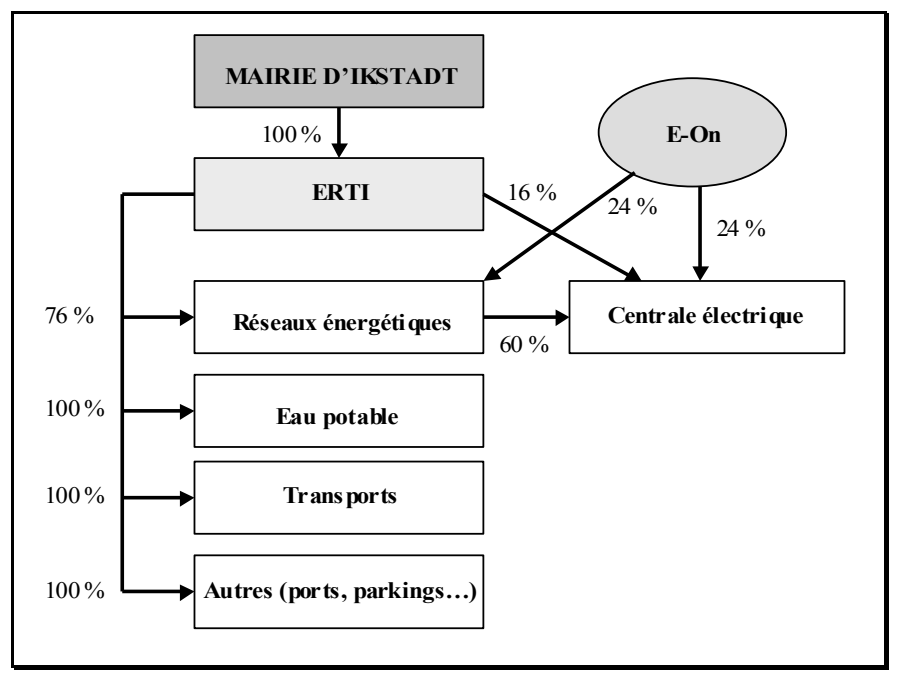

\subsection{Le marché, un puissant opérateur de recomposition du système de pilotage}

Conjuguée à l'ouverture la concurrence, la pression accrue sur les finances locales n'est pas sans conséquence sur la nature des relations qu'entretiennent ERTI et sa ville partenaire. De prime abord, l'impression générale peut être celle d'un certain durcissement, du fait, depuis quelques années, de pressions de plus en plus importantes exercées par la ville sur l'entreprise. Après s'être contentée, pendant longtemps, de la compensation du déficit des transports et du paiement des redevances de concession et d'occupation du domaine public, la municipalité 
accroît ses exigences en matière financière. Depuis 1998, elle demande à toucher des dividendes, ce qu'elle n'avait jamais fait auparavant, ponctionnant ainsi - si l'on compte la résorption du déficit des transports - près de $15 \%$ du CA d'ERTI. Ce signal fort, qui intervient à un moment où la libéralisation bouscule déjà beaucoup d'habitudes en interne, entretient un climat de tension dans l'entreprise, qui craint que la ville ne soit tentée de vendre encore tout ou partie de son capital.

\section{L'autre face de la contrainte : autonomisation et mise à distance du politique}

Paradoxalement pourtant, cette évolution reste bien vécue par l'équipe dirigeante du Stadtwerk. En effet, tout en étant porteuse d'incertitudes et de remises en cause, la contrainte concurrentielle et financière joue également dans le sens d'une plus grande autonomisation du Stadtwerk vis-à-vis de sa tutelle historique. Les exigences accrues de la municipalité - et d'E-On - se retournent aussi contre le politique et contre certaines de ses demandes qui auraient trouvé un écho plus favorable dans le contexte du monopole. Lorsque la ville demande à ERTI une participation financière à un projet d'urbanisme, la mise en place d'une nouvelle ligne de bus non rentable ou l'appel à un architecte de renom pour la réfection de la centrale électrique, il est beaucoup plus aisé pour les dirigeants d'ERTI de brandir la menace que pourraient faire peser ces projets parfois dispendieux sur la rentabilité de l'entreprise. En montrant aux élus qu'ils ne peuvent pas obtenir de leur Stadtwerk «le beurre et l'argent du beurre » et en les plaçant ainsi devant leurs responsabilités, les dirigeants d'ERTI parviennent ainsi, dans de nombreux cas, à bloquer les demandes politiques.

S'il se fait sentir dans l'ensemble des branches ${ }^{12}$, ce changement est surtout sensible dans l'énergie, qui est la plus exposée au processus de libéralisation. La contrainte de l'ouverture à la concurrence est mobilisée de trois manières par les dirigeants de la branche. Ceux-ci mettent tout d'abord en avant un "impératif » auquel on peut difficilement s'opposer : avoir davantage de marges de manœuvre dans la gestion de l'activité, au risque sinon de ne pouvoir lutter à armes égales avec des concurrents présumés non bureaucratiques et réactifs vis-à-vis du marché. Ainsi donc, la ville accepte de desserrer un certain nombre de contrôles qu'elle exerçait par le passé, notamment sur les tarifs de l'électricité, désormais librement fixés par ERTI, ce qui constitue une véritable révolution ${ }^{13}$. La présence d'E-On dans le capital de l'entreprise constitue une autre ressource pour les dirigeants de l'entreprise. De par la minorité de blocage dont il dispose, l'énergéticien fait en effet valoir des intérêts et des considérations qui sont

\footnotetext{
${ }^{12}$ même dans les transports, dans un contexte où la perspective d'une plus forte autonomisation est évoquée avec insistance, la rhétorique gestionnaire gagne en légitimité et permet aux dirigeants, plus que par le passé, de s'opposer à certains projets coûteux

${ }^{13}$ en fait, deux tarifs coexistent, l'un, dit réglementé, dont la fixation dépend de l'Etat fédéral, l'autre, dit libre, laissé à la seule appréciation du Stadtwerk. Le client final a aujourd'hui le choix entre ces deux tarifs, sachant toutefois que le tarif libre est souvent plus intéressant pour lui à court terme. En tout état de cause, dans les deux cas, la ville n'intervient plus dans le processus décisionnel
} 
souvent congruents avec ceux des dirigeants d'ERTI car fondés, eux aussi, sur une logique industrielle et financière. Son entrée au capital et sa participation aux instances délibératives rompent le huis clos des relations héritées du passé, remettent en question l'opacité de certaines demandes qui doivent être explicitées, motivées, au risque sinon d'être purement et simplement écartées. L'affirmation de plus en plus forte du Land et de l'Etat fédéral dans la régulation du secteur énergétique constitue enfin une dernière arme, tout aussi efficace, aux mains des dirigeants d'ERTI pour contenir les demandes de la ville. Jusqu'à la veille de la libéralisation, ces deux administrations ne disposaient que de pouvoirs réduits, laissant d'importantes marges de manœuvre aux communes. L'Etat fédéral était essentiellement impliqué dans les questions de production et de transport. Quant au Land, il exerçait, certes, un contrôle de l'activité du Stadtwerk, afin notamment de vérifier l'absence de transferts financiers indus en direction de la ville. Toutefois, ce rôle n'avait qu'une portée limitée et il n'avait guère d'impact sur le pilotage de la branche énergie et le contrôle des coûts. Avec la libéralisation, la donne change considérablement et se traduit par une immixtion croissante de ces deux échelons. Dans un système totalement libéralisé, où les Stadtwerke doivent désormais assurer à l'ensemble des opérateurs un accès non discriminatoire à leurs réseaux, la fixation du tarif de "péage» destiné à rémunérer le transit d'énergie devient déterminante. Elle induit un droit de regard de la nouvelle autorité de régulation sur les coûts comptabilisés par le Stadtwerk, dans la mesure où un montant trop élevé risquerait de compromettre l'arrivée de nouveaux entrants. Partant, la relation entre ERTI et la ville se trouve modifiée. L'immixtion d'un tiers acteur, l'autorité de régulation, donne de plus en plus de poids aux dirigeants du Stadtwerk, qui, brandissant la menace d'une sanction, peuvent plus facilement résister à certaines demandes émanant des élus. Ainsi donc, là encore, le marché participe activement d'une «mise à distance » ou, plus précisément, d'une recomposition de la nature de la régulation politique qui prévalait auparavant.

La plus grande autonomie dont jouissent les dirigeants d'ERTI n'est pas cependant synonyme d'éloignement progressif de deux institutions jusque là fortement imbriquées, au contraire. Entre la mairie, qui ne souhaite pas « casser sa poule aux œufs d'or », et ERTI, qui tient à préserver une qualité de relation à même de garantir son indépendance vis-à-vis des grands énergéticiens, les liens se resserrent au quotidien. Sachant jouer de son ancrage local comme d'un argument de vente vis-à-vis de ses clients, ERTI continue de participer activement à bon nombre de partenariats avec la ville. Lorsqu'elle ne les propose pas elle-même, elle répond favorablement à des projets de coopération lancés par la ville en vue de réaliser des économies budgétaires. Par exemple, des collaborations se font plus étroites en matière de gestion de la voirie, notamment sur la coordination des travaux. Dans ce type d'activités opérationnelles, bien loin d'éloigner les partenaires, le marché et le durcissement des contraintes financières agissent donc comme de puissants facteurs de coopération entre des institutions jusque là pas toujours soucieuses d' « optimiser » leurs relations. 
Un système d'interaction qui s'élargit tout en maintenant la centralité du couple maire-dirigeants

Ce poids croissant des logiques gestionnaire et financière donne en outre une importance grandissante à deux acteurs autrefois absents ou effacés du pilotage d'ERTI. Le premier est évidemment E-On. En rentrant dans le capital du Stadtwerk, l'énergéticien ne souhaite pas s'en tenir qu'à une opération purement financière, lui assurant un nouveau revenu par l'accès privilégié à la clientèle d'Ikstadt et de sa proche agglomération. Il entend également s'impliquer dans les grandes décisions stratégiques prises par ERTI et par là même dépasser le simple rôle de fournisseur qui lui était auparavant dévolu. Nous nous attarderons davantage sur le second acteur, la holding, qui profite lui aussi des évolutions récentes pour se repositionner sur la scène interne. Cette montée en puissance ne se mesure pas qu'à l'aune de la croissance de ses effectifs, à une période où les branches connaissent de sérieuses restructurations. Elle ressort également de la place grandissante que la holding occupe dans le pilotage interne. Légitimée par la menace d'un désengagement possible de la ville au cas où ERTI ne saurait dégager des dividendes suffisants, la holding parvient à s'imposer plus que par le passé. Elle a davantage son mot à dire dans les programmes d'investissement et dans les arbitrages entre projets. Au nom d'une sacro-sainte réduction des coûts, son droit de regard s'accroît dans le fonctionnement interne de branches qui tenaient, jusque là, à conserver leurs prérogatives.

L'ascendant pris par ces deux acteurs, auxquels on pourrait rajouter les clients, n'est pourtant pas de nature à remettre radicalement en question la centralité du couple maire-dirigeants dans le mode de pilotage du Stadtwerk. Tout en étant plus tendue qu'auparavant, le maire manifestant sa volonté de mieux contrôler ERTI, au besoin en mobilisant la "contre expertise » d'E-On, la relation entre les deux acteurs forts du «système Stadtwerk » se resserre un peu plus. Bien que jouant avec leurs contraintes respectives afin d'obtenir davantage satisfaction de la part de l'autre partie, le maire et l'équipe de direction d'ERTI ont bien conscience qu'ils ont tout intérêt à maintenir un bloc uni. Ne souhaitant pas se séparer de son partenaire, le maire sait qu'il ne doit pas trop déstabiliser une structure qui lui apporte d'importantes ressources et qui lui permet d'effacer le déficit des transports. De leur côté, les dirigeants d'ERTI comprennent que la préservation de leur indépendance vis-à-vis des autres opérateurs - au premier rang desquels EOn, qui pourrait lorgner sur une prise de participation majoritaire - passe par le maintien d'une volonté politique de conserver telle quelle la situation. Condamnés à vivre ensemble, maire et dirigeants continuent donc d'entretenir des liens forts, dans la continuité de ceux qui existaient depuis la création d'ERTI. Le maintien de cette forte proximité se double d'un net renforcement des positions du maire et des dirigeants d'ERTI au sein de leur institution. La « contrainte objective » du marché et de la concurrence que les dirigeants d'ERTI ne cessent de renvoyer aux politiques est aussi un argument que le maire peut retourner contre certains de ses conseillers qui se font le relais de demandes de fonctionnaires territoriaux ou de la population jugées exorbitantes. De ce fait, le maire se voit renforcé dans son rôle 
d'intercesseur. Il conforte un leadership politique qui, bien qu'étant «constitutionnalisé » par la législation du Land, avait toujours été par le passé tempéré par l'existence de contre-pouvoirs au sein du conseil municipal ${ }^{14}$. Quant à eux, les dirigeants d'ERTI ne sont guère affaiblis par le poids de la holding. Bien au contraire, ils y trouvent un allié de taille pour imposer aux branches des décisions qu'il leur était parfois difficile de faire passer précédemment, en matière de satisfaction des clients, de prise en considération de certaines demandes de la ville ou de rationalisation des coûts. Ainsi donc, tout en ouvrant davantage le jeu des relations internes, les évolutions macro-économiques affectant le «système ERTI » ne sauraient être interprétées dans le sens d'une rupture par rapport au passé.

\section{Conclusion}

Tout comme l'Etat est conduit, en France et dans les pays où il s'est vu conférer un rôle central dans la gestion du service public, à s'approprier de nouvelles fonctions, les collectivités locales allemandes apparaissent aujourd'hui comme le lieu d'apprentissage de ce que nous pouvons appeler un nouveau mode de gouvernement local des réseaux. Dans un cas comme Ikstadt, qui représente la «voie médiane» suivie par beaucoup de villes, les pressions financière et concurrentielle ne jouent pas pour le moment - et ce malgré certaines prophéties dans le sens d'une éradication pure et simple de l'intervention municipale, marquant la fin programmée d'un modèle de gestion du service public original. Elles induisent plutôt une reconfiguration des relations très étroites que la ville et ERTI ont toujours entretenues depuis le développement des premiers réseaux urbains.

L'examen approfondi du cas d'Ikstadt nous donne à voir deux éléments fondamentaux de cette refondation. Le premier touche aux formes même de l'exercice du pouvoir politique, avec le passage d'une relation de tutelle souvent discrétionnaire à un mode d'interaction beaucoup plus marqué par la formalisation. Jusqu'au tournant des années 1990, la protection dont bénéficiait ERTI vis-à-vis du marché (situation de monopole) et de son actionnaire public (faibles exigences en matière de dividendes) constituait, dans le même temps, une contrainte forte dans les relations que le Stadtwerk entretenait avec la ville. En effet, arguant de la situation privilégiée d'ERTI, lorgnant sur une structure considérée comme plus riche que les services municipaux, fonctionnaires et élus étaient toujours tentés de faire pression sur les différentes branches. Malgré le poids des dirigeants mais aussi du maire, qui disposait de pouvoirs plus importants que sur le reste du territoire allemand, il n'était pas toujours aisé de résister trop fortement à certaines demandes, surtout lorsqu'elles étaient relayées

\footnotetext{
${ }^{14}$ comme le souligne Hellmut Wollmann, le caractère «présidentiel » du gouvernement local dans les Länder du sud de l'Allemagne ne virait jamais à l'autocratie, du fait d'un système de checks and balance qui obligeait le maire à tenir compte des positions des conseillers municipaux (Wollmann, 2004)
} 
par une partie de la population locale. En véhiculant de nouvelles valeurs d'action publique, en créant et naturalisant de nouvelles contraintes pour les opérateurs, les réformes néo-libérales qui traversent le secteur énergétique et le mode de gestion des villes allemandes participent de ce que nous avons appelé une «mise à distance » de ce type de demandes politiques. Lorsqu'il faut faire des économies, lorsqu'il faut justifier ses coûts auprès du Land et de l'autorité de régulation, le marché devient le meilleur allié des techniciens. "Tiers acteur» venant briser la relation de huis clos qui s'était précédemment instaurée, il permet de résister à certaines demandes locales, jugées exorbitantes, d'être dans un rapport de forces plus favorable vis-à-vis d'élus et de fonctionnaires qui pourraient être tentés d'abuser de leur position. Ceci étant, les relations ne disparaissent pas à proprement parler. Elles se formalisent plus que par le passé. Aux arrangements opaques succèdent des demandes plus explicites. Elles prennent la forme de contrats ou de partenariats ponctuels. Elles précisent plus que par le passé les attentes que la ville peut avoir envers son opérateur, attentes qui autrefois restaient souvent dans le registre de l'implicite.

Ce processus de «mise à distance » du politique et la formalisation croissante qui l'accompagne ont une autre conséquence majeure. Tout comme elle contribue à recomposer les rapports de force au sein du Stadtwerk, la logique gestionnaire prenant peu à peu le pas sur une rationalité technique longtemps prédominante, la référence au marché induit de nouveaux équilibres dans l'administration municipale. Déjà confortés dans leur rôle par la crise budgétaire communale, les financiers occupent un poids plus important. De même, le maire sort renforcé par la «mise à distance » du politique, qui touche surtout les conseillers municipaux porteurs des demandes locales ou attachés à la gestion de certaines activités ${ }^{15}$. Cette sensibilité plus grande à la logique financière, cette plus forte centralisation du système décisionnel, qui se doublent d'une diversification de l'expertise jusque là monopolisée par le Stadtwerk, participent d'une profonde transformation de l'exercice du pouvoir politique, non de son éradication pure et simple. Dans un contexte où les réformes tendent partout à renforcer les logiques d'intégration sectorielle au détriment de l'intégration territoriale, celui-ci se déplace, il se reconstitue pour évoluer vers une fonction de pilotage stratégique et financier que la période précédente, celle du monopole et de la prospérité communale, avait tendance à minorer. Cette mutation importante, dont les conséquences sont encore à découvrir, montre bien la nécessité de réintégrer pleinement le rôle du politique et de l'exécutif local dans les analyses sur la gouvernance urbaine (Le Galès, 2003).

\footnotetext{
${ }^{15}$ on peut penser que ce poids croissant du maire ressortirait beaucoup plus nettement dans le cas de villes où le régime de type « présidentiel » n'existait pas précédemment
} 


\section{BIBLIOGRAPHIE}

Brunekreeft, G., Keller, K., 2000. The electricity supply industry in Germany : market power or power of the market ? Utilities Policy (9), 15-29.

Cohen, E., 1992. Le Colbertisme "high tech". Economie des Telecom et du Grand Projet. Hachette, Paris.

Courivaud, H., 2000. Le droit de la concurrence contre les libertés communales ? L'exemple des Stadtwerke allemandes confrontées à la libéralisation du secteur de l'électricité. Revue Internationale de Droit Economique 14 (3), 405-439.

Eberlein, B., 2000. Institutional Change and Continuity in German Infrastructure Management: The Case of Electricity Reform. German Politics 9 (3), 81-2004.

Eising, R., 1999. Reshuffling Power. The liberalisation of the EU electricity markets and its impact on the German governance regime. In : Kohler-Koch, B., Eising, R., 1999. The transformation of Governance in the European Union. Routledge, London.

Jamous, H., 1967. Contribution à une sociologie de la décision. La Réforme des études médicales et des structures hospitalières. CES, CNRS, Paris.

Le Galès, P., 2003. Le retour des villes européennes. Presses de Sciences Po, Paris.

Levi-Faur, D., 1999. The Governance of Competition: the interplay of technology, economics, and politics in European Union electricity and telecom regimes. Journal of Public Policy 19 (2), 175-207.

Lorrain, D., 2002a. Capitalismes urbains. Des modèles européens en compétition, L’Année de la régulation (6), 2002-2003, 195-239.

Lorrain, D., 2002b. Le modèle public local fort. Les entreprises de réseaux techniques urbains en Allemagne et aux Pays-Bas. Rapport au Commissariat Général du Plan, Paris.

Marcou, G., 1995. Les modes de gestion des services publics locaux en Allemagne et l'ouverture à la concurrence. Revue Française de droit administratif 11 (3).

Muschen, K., 1993. Kommunale Energiepolitik. In : Roth, R., Wollmann, H. (Ed.). Kommunalpolitik. Politisches Handeln in den Gemeinden Bundeszentrale für politische Bildung, Schriftenreihe Band 320, Bonn. 
Schmidt, S. K., 1997. Sterile Debates and Dubious Generalisations : European Integration Theory Tested by Telecommunications and Electricity. Journal of Public Policy 16 (3), 233-271.

Steckert, U., 2002. Liberalization, Competition and Identity Crisis in Municipal Enterprise. Deutsche Zeitschrift für Kommunalwissenschaften 41 (1).

Wollmann, H., 2002. Is Germany's Traditional Type of Local Self-Government Being Phased out? Deutsche Zeitschrift für Kommunalwissenschaften 41 (1).

Wollmann, H., 2004. Urban leadership in German Local Politics : The Rise, Role and Performance of the Directly Elected (Chief Executive) Mayor. International Journal of Urban and Regional Research 28 (1), 150-165. 
Annexe. Quelques données sur ERTI (2002)

\begin{tabular}{|l|c|c|c|}
\cline { 2 - 3 } \multicolumn{1}{c|}{} & $\begin{array}{c}\text { Chiffre d'affaires } \\
\text { (millions d' } € \text { ) }\end{array}$ & Effectifs & $\begin{array}{c}\text { Bénéfices avant } \\
\text { redistributions } \\
\text { diverses } \\
\text { (millions d' } € \text { ) }\end{array}$ \\
\hline ERTI & 216 & $1135^{16}$ & $\mathbf{2 , 8}$ \\
\hline Energie (STW) : & 163 & 414 & 17,9 \\
\hline Eau potable & 19 & 39 & $\mathbf{0}$ \\
\hline Transports & 22,3 & 316 & $\mathbf{1 7 , 2}$ \\
\hline Port & $\mathbf{2}$ & 12 & $\mathbf{0 , 4}$ \\
\hline Gestion des déchets & $\mathbf{9 , 8}$ & 21 & $\mathbf{0 , 5}$ \\
\hline Autres (parkings...) & 17 & 21 & \\
\hline
\end{tabular}

${ }^{16}$ les effectifs correspondent à ceux des différentes filiales d'ERTI auxquels se rajoutent les personnes travaillant dans la holding 